\title{
Folgen der postpartalen Hämorrhagie und optimale Nachbetreuung der Frau
}

Ines Neukom, Hebamme BSc, Zürich

\section{Verschiedene Studien belegen die steigenden Raten an postpartalen Hämorrhagien (PPH). Der massive Blutverlust stellt ein schwerwiegendes Ereignis dar und kann zu tiefgreifenden physischen und psychischen Folgen führen, in gravierenden Fällen sogar tödlich verlaufen. In ihrer Bachelorarbeit stellt unsere Autorin anhand evidenz- basierter Literatur die Folgen einer PPH dar und legt Empfehlungen für die optimale Nachbetreuung der betroffenen Frau vor.}

Dieser Beitrag basiert auf einer wissenschaftlichen Arbeit, die mit dem 2. Bübchen Wissenschaftspreis für Hebammen prämiert wurde.

Originalarbeit: Neukom Ines (2015). Die postpartale Hämorrhagie (PPH) - ihre physischen und psychischen Folgen auf die betroffene Frau und deren optimale Nachbetreuung. Bachelorarbeit an der Zürcher Hochschule für Angewandte Wissenschaften (ZHAW), Departement Gesundheit, Institut für Hebammen

\section{Hintergrund}

„Ich verlor in Kürze so viel Blut... und ich konnte mich selbst sterben fühlen ..."

(durch Autorin übersetzt aus Elmir, Schmied, Wilkes [9])

Mit diesen bewegenden Worten erinnert sich eine Frau an die dramatischen Momente nach der Geburt ihres Kindes zurück, bei der sie aufgrund eines gravierenden Blutverlusts beinahe gestorben wäre.

Eine postpartale Hämorrhagie (PPH) ist eine lebensbedrohliche Geburtskomplikation und weltweit eine der Hauptursachen mütterlicher Morbidität und Mortalität [21]. Die Prävalenz in den Industrienationen beträgt rund 0,5-5\% aller Geburten [30]. Die PPH-Rate hat sich laut einer Studie in Kanada in den Jahren 2000 bis 2009 um 27\% erhöht [18]. Ein möglicher Grund dafür könnte die steigende Zahl an Geburtseinleitungen sein, die einen Risikofaktor für eine PPH darstellen. Mit steigender Tendenz der PPHRate gewinnt das Thema an Bedeutung.

Eine PPH kann tödlich verlaufen. Und wenn sie nicht zum Tod führt, kann sie schwerwiegende körperliche sowie psychische Folgen nach sich ziehen. Dies wiederum kann negative Auswirkungen auf die Familie der betroffenen Frau haben [5]. Die physischen und psychischen
Folgen einer PPH verdienen deshalb entsprechend hohe Aufmerksamkeit in der Geburtsbegleitung.

\section{Prävalenz}

Die Prävalenz für eine PPH in den Industrienationen beträgt ca. 0,5-5\% aller Geburten [30]. Weltweit liegt sie im Durchschnitt bei ca. 6\%, wobei Afrika die höchste Rate verzeichnet mit 10,5\% [6]. Die PPH zählt zu den Hauptursachen mütterlicher Morbidität und Mortalität [30]. Weltweit sterben jährlich ca. 150000 Frauen aufgrund einer PPH [13].

\section{Definition PPH}

Um die PPH in ihrer Ausprägung klarer zu erfassen, dienen folgende Definitionen aus der Literatur [14]:

Eine postpartale Hämorrhagie (PPH) wird von der WHO [34] definiert als

- Blutverlust > $500 \mathrm{ml} / 24$ Std. nach vaginaler Geburt

- Blutverlust > $1000 \mathrm{ml} / 24$ Std. nach Kaiserschnitt

Eine schwere Blutung wird definiert als

- Blutverlust > $150 \mathrm{ml}$ pro Min. innerhalb von 20 Min.

- Verlust von 50\% des zirkulierenden Blutvolumens innerhalb von 3 Std.

- oder akuter Blutverlust > 1500 - 2000 ml / 24 Std.

Eine PPH kann primär (früh) also in den ersten 24 Stunden postpartum oder sekundär (spät) ab 24 Stunden postpartum bis 12 Wochen postpartum auftreten [28]. 
Tab. 1 Risikofaktoren für eine postpartale Hämorrhagie.

\begin{tabular}{|c|c|}
\hline Zeitraum & Risikofaktoren \\
\hline antepartal & $\begin{array}{l}\text { - Abruptio placentare } \\
\text { - Placenta praevia } \\
\text { - Status nach Sectio caesarea } \\
\text { - Überdehnung des Uterus (Mehrlingsschwangerschaften, } \\
\text { Polyhydramnion, Makrosomie) } \\
\text { - Multiparität (> 4) } \\
\text { - Präeklampsie } \\
\text { - Status nach Retentio placentae } \\
\text { - Status nach postpartaler Hämorrhagie } \\
\text { - Präpartales/maternales Hämoglobin < 8,5 g/dl oder 5,28 mmol/l } \\
\text { - BMI> } 35 \text { kg/m² } \\
\text { - Maternales Alter (> } 35 \text { Jahre) } \\
\text { - Uterusanomalien } \\
\text { - Plazentare Insertionsstörungen (accreta, increta, percreta) }\end{array}$ \\
\hline intrapartal & $\begin{array}{l}\text { - Geburtseinleitung } \\
\text { - Prolongierte Geburtsphasen } \\
\text { - Einsatz von Oxytocin } \\
\text { - Vaginal-operative Geburt } \\
\text { - Sectio caesarea (insbesondere sekundäre Sectio caesarea) }\end{array}$ \\
\hline
\end{tabular}

nach Hösli et al. [15]
In den Industrienationen beträgt das Risiko, an einer PPH zu sterben, ca. $1: 100000$, in Entwicklungsländern $1: 1000$ Geburten [20]. Die Geburtseinleitung stellt einen Risikofaktor für eine PPH dar [15]. Nach einer Geburtseinleitung mit Prostaglandinen oder Oxytocin ist die Rate an PPH signifikant erhöht [17].

\section{Risikofaktoren}

In Tab. 1 werden die ante- sowie die intrapartalen Risikofaktoren für eine PPH dargestellt.

\section{Ursachen und Diagnose}

Die möglichen Ursachen einer PPH werden im angloamerikanischen Raum über die 4 Ts (Tonus, Trauma, Tissue, Thrombin) definiert. Die Ursachen können da-

Tab. 2 Ursachen der postpartalen Hämorrhagie - die 4 Ts und ihre Diagnose.

\begin{tabular}{|l|l|l|l|l|}
\hline & T & Ursache & Anteil (\%) & Diagnose \\
\hline 1 & Tonus & Uterusatonie & $70-90$ & Höhenstand und Uteruskonsistenz \\
\hline 2 & Trauma & Weichteilverletzung, Uterusruptur & 20 & Inspektion der Geburtswege \\
\hline 3 & Tissue & $\begin{array}{l}\text { Plazentaretention, Plazentalösungs- } \\
\text { störung, Plazentaimplantationsstörung }\end{array}$ & 10 & Vollständigkeit der Plazenta überprüfen (visuell, \\
\hline 4 & $\begin{array}{l}\text { Thrombin } \\
\text { (Koagulopathie) }\end{array}$ & $\begin{array}{l}\text { Primär traumatisch (bei hohem Blut- } \\
\text { verlust aus 1-3) } \\
\text { Primär koagulopathisch }\end{array}$ & ca. 1 & $\begin{array}{l}\text { Klinisch: z. B. diffuse Blutung, Blut gerinnt nicht } \\
\text { Labor: Gerinnungsstatus, Blutbild ROTEM* } \text {, TEG ** } \\
\text { * Rotationsthrombelastometrie } \\
\text { ** Thrombelastografie }\end{array}$ \\
\hline
\end{tabular}

Schlembach et al. [25], modifiziert nach Rath und Bohlmann [23]

Tab. 3 Behandlungsschritte bei PPH.

\begin{tabular}{|c|c|}
\hline Ursache & Behandlungsschritte \\
\hline Trauma & Rasche chirurgische Versorgung der Weichteilverletzung oder Uterusnaht bei Uterusruptur. \\
\hline Thrombin & Substitution der Gerinnungsfaktoren. \\
\hline
\end{tabular}

Diese Maßnahmen werden vom Expertenbrief Nr. 26 der Schweizerischen Gesellschaft für Gynäkologie und Geburtshilfe (SCGG) von Surbek et al. [30] gestützt. 
bei kombiniert oder auch sequenziell auftreten [25]. Tab. 2 stellt die Ursachen, ihren prozentualen Anteil sowie die Diagnosemöglichkeiten dar.

\section{Therapiemethoden}

Die Therapie einer PPH besteht darin, die Blutungsursache zu beheben, den Kreislauf zu stabilisieren sowie eine Gerinnungsstörung zu behandeln [23]. Die Behandlung einer PPH erfolgt je nach Blutungsursache unterschiedlich. Dazu werden in Tab. 3 die 4 Ts und die dazugehörenden Behandlungsschritte aufgeführt.

\section{Physische und psychische Folgen für die Frau}

Die Literaturanalyse ergab, dass eine PPH schwerwiegende physische wie auch psychische Folgen für die betroffene Frau haben kann.

\section{Physische Folgen für die Frau}

Nach einer PPH leiden Frauen tendenziell eher an einer durch den erhöhten Blutverlust bedingten Eisenmangelanämie [16]. Diese kann verschiedene, zum Teil schwerwiegende Konsequenzen nach sich ziehen. Stellvertretend seien erwähnt: körperliche Erschöpfung, Infektionen [16], verminderte Milchproduktion [7], emotionale Labilität und ein erhöhtes Risiko für eine Postpartale Depression (PPD) $[3,8,19]$.

Auch Stillprobleme können eine gravierende Folgeerscheinung der PPH sein. Durch die als Folge einer schweren PPH entstehende akute Notfallsituation ist es meist nicht möglich, ein frühzeitiges Anlegen an die Brust zu gewährleisten. Je später das erste Anlegen stattfindet, desto weniger gelingt später das Stillen [31]. Tiefere Stillraten und eine kürzere Stilldauer können die Folge sein [31]. Eine reduzierte Laktation ist ein weiteres Problem. Sie entsteht durch Eisen- mangelanämie [7] oder durch stressbedingt erhöhte Cortisolwerte [12]. Die beschriebenen physisch verursachten Stillprobleme können auch psychische Probleme nach sich ziehen [31].

Eine längere postpartale Blutungsdauer, ein erhöhtes Risiko für uterine Infektionen sowie eine erhöhte Rehospitalisierungsrate aufgrund von Mastitis, Abszessen, erneuten Blutungen, Infektionen sowie tiefen Hämoglobinwerten sind weitere physische Folgen der PPH [32].

In sehr seltenen Fällen kann es nach einer schweren Blutung, bedingt durch die Ischämie der Hypophyse, zum Sheehan-Syndrom kommen. Es handelt sich dabei um eine akute Hypophyseninsuffizienz, die meist den Hypophysenvorderlappen betrifft. Dies kann zu einem ACTH Mangel, Nebennierenfunktionsstörungen und in Folge dessen zu schweren hormonellen Veränderungen führen. Klinisches Leitsymptom ist zunächst die fehlende Laktation postpartum. Weitere Symptome, die sich zum Teil auch erst Jahre später zeigen, sind Amenorrhöe, sekundäre Infertilität, Erschöpfung, Hypoglykämie, Ausfall der sekundären Körperbehaarung und verminderte Libido $[10,22,26]$.

\section{Psychische Folgen für die Frau}

In einer akuten Notfallsituation - wie sie eine PPH darstellt - werden die individuellen Informationsbedürfnisse der Frau häufig übergangen und die Kommunikation mit der Frau und ihrem Partner tritt in den Hintergrund. Frauen nach einer PPH haben ein großes Informationsbedürfnis, leider wird darauf häufig nicht genügend eingegangen [29, 33]. Frauen, bei denen dieses Bedürfnis nicht befriedigend gestillt wurde, fühlen sich entmachtet, übergangen, hilflos, verletzt, verängstigt und/oder wütend [29]. Unterlassen die Fachpersonen das adäquate Informieren der Frau während und nach der PPH, kann dies die Verarbeitung und Akzeptanz des fatalen Ereignisses erschweren [29].
Aus mehreren der analysierten Studien geht hervor, dass eine PPH traumatisierend sein kann $[9,29,33]$. Ein Großteil der in den Studien $[9,27]$ untersuchten Frauen empfand während der PPH Todesangst. In beiden Studien berichteten die Frauen, in Flashbacks das Trauma wieder zu durchleben. Bei einem Teil der Frauen bestand auch nach dem Ereignis weiterhin persistierende Angst [27]. Die Angst vor Kontrollverlust [9] sowie die Entwicklung von Krankenhaus-, oder Blutphobien sind weitere mögliche Folgen einer PPH [27].

Körperliche Primärfolgen wie Eisenmangelanämie und körperliche Erschöpfung können als psychische Sekundärfolge zu einer Postpartalen Depression (PPD) führen, denn beides sind Risikofaktoren für eine PPD [8]. Eine PPD tritt zudem häufiger nach komplizierten Geburtsverläufen auf [11]. Aus diesen Erkenntnissen lässt sich folgern, dass nach einer PPH das Risiko für eine PPD erhöht ist. Des Weiteren haben Frauen nach einer traumatischen Geburt ein erhöhtes Risiko, eine Posttraumatische Belastungsstörung (PTSD) zu entwickeln [1, 4, 24].

Eine PPH kann potenziell langfristige psychologische Folgen für die Frau haben.

\section{Folgen für die Mutter-Kind- Beziehung}

Bedingt durch die akute Notfallsituation während einer PPH kann in vielen Fällen das Bonding erst später erfolgen. Dies stellt einen suboptimalen Start für die Mutter-Kind-Beziehung dar. Die körperliche Trennung vom Kind und das verspätete Bonding kann auf die Frau belastend wirken [27, 33].

Die zuvor beschriebene Eisenmangelanämie und die körperliche Erschöpfung der Frau können weitere negative Auswirkungen auf den Beziehungsaufbau zwischen Mutter und Kind haben. Bedingt durch die körperliche Schwächung ist es den Frauen in der ersten Zeit post- 
partum oft nicht möglich, ihr Kind selbstständig zu versorgen [33]. Dies kann zu Selbstvorwürfen, Versagensgefühlen sowie psychischem Stress führen. Diese Gefühle können zudem verstärkt werden, wenn bedingt durch die beschriebene reduzierte Laktation Stillprobleme auftreten.

\section{Folgen für Familie und Partnerschaft}

Eine PPH kann außerdem negative Folgen für die sexuelle Aktivität haben [27], was wiederum die Beziehung zum Partner negativ beeinflussen kann [2].

Aus Angst vor einer Wiederholung der PPH verzichten Frauen häufig auf eine Folgeschwangerschaft oder schieben diese hinaus $[27,32,33]$. Dies kann für ein Paar sehr belastend sein und mitunter die ganze Familie beeinflussen.

Frauen, die nach einer PPH eine Folgeschwangerschaft austragen, haben ein großes Risiko, während dieser Schwangerschaft starke Angstgefühle zu entwickeln [27].

\section{Interaktion der Folgen}

Aus der herangezogenen Literatur geht hervor, dass die einzelnen Folgen der PPH zum Teil stark miteinander zusammenhängen, sich gegenseitig beeinflussen und sich sogar verstärken können. Dies lässt sich beispielsweise durch folgende Situation veranschaulichen (Abb.1): Aufgrund der körperlichen Erschöpfung ist es der Mutter oft nicht möglich, ihr Kind ausreichend zu versorgen. Das kann dazu führen, dass das Kind unruhig wird und vermehrt schreit. Dies kann psychischen Stress bei der Mutter auslösen und sich negativ auf die Laktation auswirken. Eine Folge davon können Selbstvorwürfe sein, z.B.: „Ich bin eine schlechte Mutter." Dies wiederum verstärkt den psychischen Stress und führt damit in einen Teufelskreis. Solche Teufelskreise gilt es, gezielt zu durchbrechen. Genau hier finden sich Ansatzpunkte für die Arbeit der Hebamme.

\section{Empfehlungen für die Nachbetreuung}

Aus den Erkenntnissen der Studienanalyse lassen sich detaillierte Empfehlungen für die Praxis ableiten.

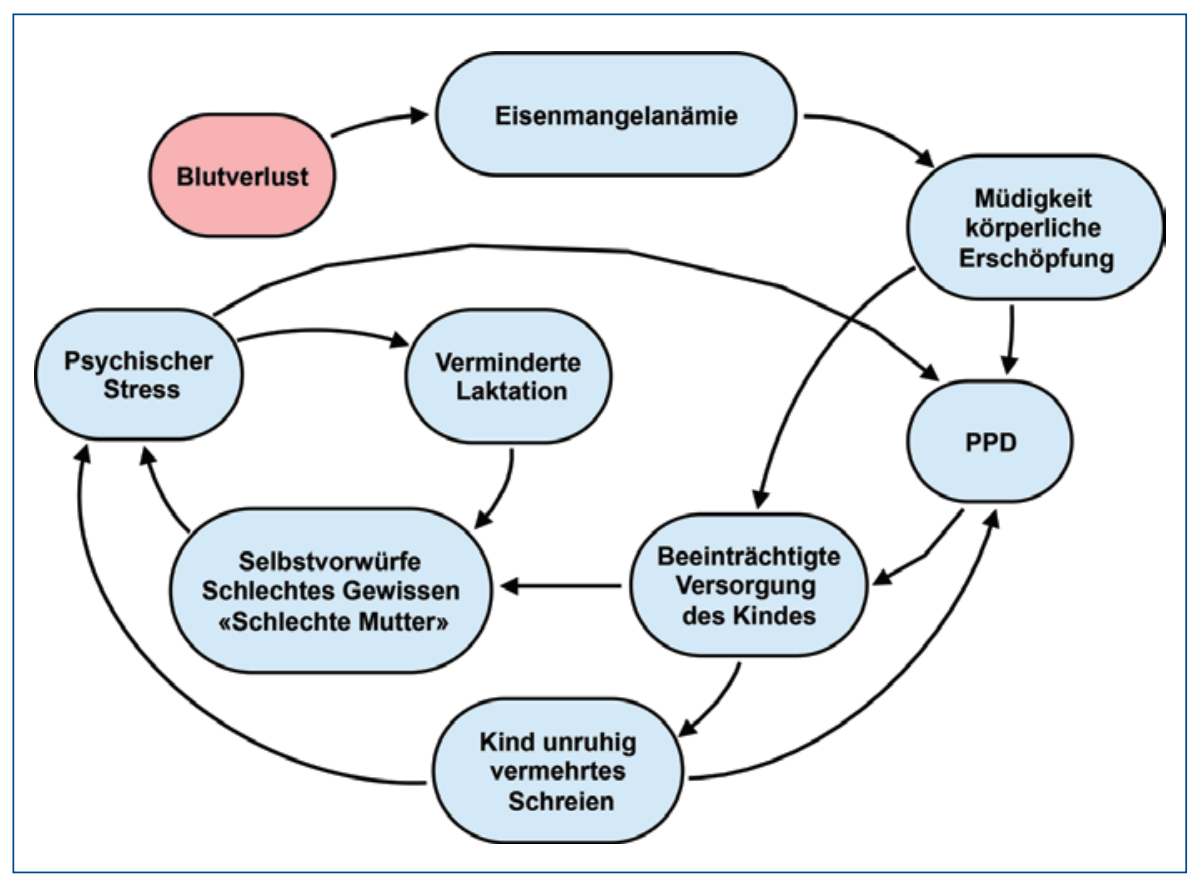

Abb. 1 Physische Primärfolge der PPH mit psychischen und physischen Sekundärfolgen (Darstellung: Ines Neukom).

\section{Information und Beratung der Frau}

Im Zentrum steht dabei das adäquate Informieren der Frau über die Ursache der PPH, die ergriffenen therapeutischen Maßnahmen sowie über die potenziellen physischen und psychischen Folgen in der Zeit postpartum. Weiter soll eine Aufklärung über die möglichen Konsequenzen hinsichtlich einer Folgeschwangerschaft und zum Wiederholungsrisiko einer PPH erfolgen. Zudem sollen der Frau Strategien aufgezeigt werden, welche die physische Genesung unterstützen. Wichtig ist, das individuelle Informationsbedürfnis der Frau zu berücksichtigen. Es sollten einfache Informationen abgegeben werden, soweit dies die Frau wünscht. Für die Betroffene sollte langfristig die Möglichkeit bestehen, Ansprechpartner für ihre Fragen zu haben.

\section{Unterstützung für den Alltag}

Die Frau sollte im Alltag mit ihrem Kind speziell unterstützt und entlastet werden. Dabei soll eine individuelle Ressourcenabklärung erfolgen. Weiter sollte dem Beziehungsaufbau zwischen Mutter und Kind besonders große Aufmerksamkeit geschenkt werden und die Frau sollte beim Bonding unterstützt werden. Bei bestehendem Stillwunsch ist es wichtig, ein erstes Anlegen an die Brust frühzeitig zu fördern sowie der Frau besonders viel Unterstützung beim Stillen zu gewähren. Die Akzeptanz für potenzielle Stillschwierigkeiten gilt es zu erhöhen und vorhandene Schuld- oder Versagensgefühle abzubauen. Dies kann erreicht werden, indem der Frau die pathophysiologischen Zusammenhänge zwischen einer PPH und Stillschwierigkeiten erklärt werden.

\section{Medizinische Behandlung}

Um den durch eine etwaige Eisenmangelanämie bedingten körperlichen Erschöpfungszuständen vorzubeugen, sollte eine 


\section{Umfrage unter Kliniken}

Die Bachelorarbeit zeigt, welche Folgen eine PPH haben kann und welche Maßnahmen Fachpersonen ergreifen können, um diese Folgen möglichst gering zu halten. Es stellte sich die Frage, inwieweit Leitlinien zur Nachbetreuung von Frauen nach einer PPH in der Praxis heute bereits existieren.

Zur Klärung dieser Frage führte die Autorin eine nicht repräsentative Umfrage bei den geburtenstärksten Kliniken der Deutschschweiz durch, welche folgendes Resultat ergab: Alle der befragten Kliniken $(n=11)$ gaben auf Nachfrage an, über keine schriftlichen Leitlinien zu verfügen, die den Umgang mit Frauen nach einer PPH regeln. Mehrere der angefragten Kliniken bekundeten, dass Bedarf an solchen Leitlinien bestünde. Einige erwähnten, dass auf ihrer Wochenbettabteilung jede Frau nach ihrem körperlichen und emotionalen Wohlbefinden aktiv befragt, ihr Unterstützung angeboten und sie auf die physischen und psychischen Folgen einer PPH hingewiesen werde. Dieses Vorgehen ist jedoch bei keinem der angefragten Spitäler schriftlich festgehalten.

Entsprechende, schriftlich festgehaltene Leitlinien könnten einen wertvollen Beitrag zur Vermeidung oder wenigstens Verminderung psychischer und physischer Folgen der PPH leisten. Betroffenen Frauen würde viel Leid erspart und zudem könnten Gesundheitskosten eingespart werden. Die Autorin entwarf aus diesem Grund eine Checkliste (Abb.2), die den Umgang mit Frauen nach einer $\mathrm{PPH}$ in den ersten Stunden und Tagen sowie nach Klinikaustritt regelt. Diese Checkliste kann inter- professionell genutzt werden und dient gleichzeitig zur Übersicht und Dokumentation, welche Informationen abgegeben und welche Interventionen

durchgeführt wurden. Die Checkliste basiert auf den Erkenntnissen, die aus der aufgeführten Literatur extrahiert wurden.

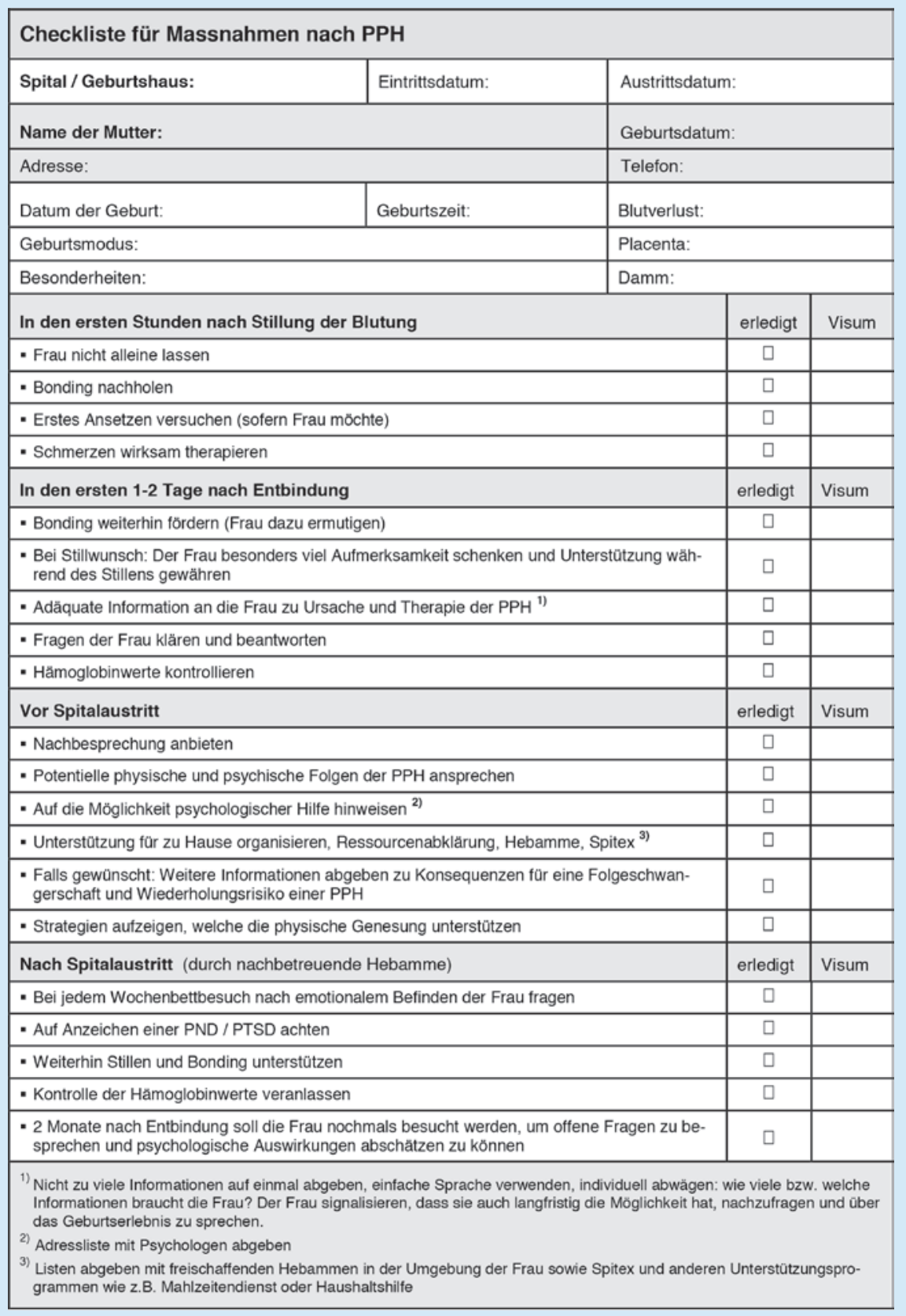

Abb. 2 Checkliste für Maßnahmen nach PPH. Quelle: Neukom Ines (2015). Die postpartale Hämorrhagie (PPH) - ihre physischen und psychischen Folgen auf die betroffene Frau und deren optimale Nachbetreuung. Bachelorarbeit an der Zürcher Hochschule für Angewandte Wissenschaften (ZHAW), Departement Gesundheit, Institut für Hebammen 
solche Anämie frühzeitig erkannt und mittels Eisensubstitution ärztlich behandelt werden.

\section{Betreuung der Frau im Status nach PPH}

Jeder Frau nach einer PPH sollte ein Nachgespräch angeboten werden. Die erste Nachbesprechung sollte innerhalb der ersten vier Wochen postpartum stattfinden [5]. Es wird empfohlen, dass die Hebamme die Frau zwei Monate postpartum nochmals besucht, um Fragen klären und die psychologischen Auswirkungen abschätzen zu können [27]. Zudem sollte die Frau auf eine psychologische Betreuung während der postpartalen Zeit hingewiesen werden. Im Rahmen der Wochenbettbesuche sollte die Hebamme gezielt nach dem emotionalen Befinden der Frau fragen.

Für die Schwangerschaftsbetreuung einer Frau im Status nach PPH ist Folgendes zu beachten: Die Aufarbeitung des Erlebnisses einer PPH sollte möglichst vor einer Folgeschwangerschaft erfolgen. Es ist wichtig, die Ängste der Frau vor einer Wiederholung einer PPH ernst zu nehmen und zu besprechen. Während der gesamten Schwangerschaftsbetreuung, ist das Risiko für die Entwicklung von starken Ängsten zu beachten. Die Frau sollte ggf. frühzeitig an eine Psychologin bzw. einen Psychologen verwiesen werden. Es sollte eine individuelle Risikoabklärung für die Wiederholung einer PPH stattfinden. Und die Frau sollte während sowie nach der Geburt angemessen überwacht werden.

\section{Umgang mit dem Partner nach einer PPH}

Selbstverständlich ist auch der Partner von einer PPH betroffen und muss deshalb ebenfalls mit in die Nachbetreuung einbezogen werden. Da der Einfluss einer PPH auf den Partner jedoch nicht Teil der Bachelorarbeit war, geht dieser Artikel nicht spezifisch darauf ein.

\section{Fazit}

Eine PPH kann schwerwiegende physische und psychische Folgen nach sich ziehen, die zum Teil stark miteinander interagieren, sich verstärken und damit in einen Teufelskreis führen können. Maßnahmen, welche die nachbetreuenden Fachpersonen zur Verminderung oder Vermeidung solcher Teufelskreise ergreifen können, sind bekannt. Für deren Umsetzung wären Leitlinien, die den Umgang mit Frauen nach PPH konkret regeln, ein gutes Hilfsmittel. Somit könnte vielen Frauen und Familien unnötiges Leid erspart bleiben.

\section{Literatur}

1 Ayers S, Pickering AD. Do women get posttraumatic stress disorder as a result of childbirth? A prospective study of incidence. Birth 2001; 28 (2): 111-118

2 Ayers S, Eagle A, Waring $\mathrm{H}$. The effects of childbirth related PTSD on women and their relationship: a qualitative study. Psychology, Health and Medicine 2006; 11 (4): 389-398

3 Beard JL, Hendricks MK, Perez EM, Murray-Kolb LE, Berg A, VernonFeagans L, Irlam J, Isaacs W, Sive A, Tomlinson M. Maternal iron deficiency anemia affects postpartum emotions and cognition. Journal of Nutrition 2005; 135: 267-272

4 Beck CT. Birth trauma: In the eye of the beholder. Nursing Research 2004; 53 (1): 28-35

5 Boyle M (2011). Emergencies around childbirth: a handbook for midwifes (2. Aufl.). London: Radcliffe Publishing

6 Carroli G, Cuesta C, Abalos E, Gulmezoglu AM. Epidemiology of postpartum haemorrhage: a systematic review. Best Practice and Research Clinical Obstetrics \& Gynaecology 2008; 22 (6): 999-1012

7 Christoph P. Eisenmangelanämie in der Schwangerschaft. Gynäkologie 2012; 2: 18-22

8 Corwin EJ, Murray-Kolb LE, Beard JL. Low hemoglobin level is a risk factor for postpartum depression. Journal of Nutrition 2003; 133: 4139-4142
9 Elmir R, Schmied V, Wilkes L. Between life and death: women's experiences of coming close to death, and surviving a severe postpartum haemorrhage and emergency hysterectomy. Midwifery 2012; 28: 228-235

10 Feinberg E, Molitch M, Endres L, Peaceman A. The incidence of Sheehan's syndrome after obstetric hemorrhage. Fertility and Sterility 2005; 84: 975-979

11 Gamble J, Creedy D. Psychological trauma symptoms of operative birth. Brithish Journal of Midwifery 2005; 13 (4): 218-224

12 Grajeda R, Perez-Escamilla R. Stress during labor and delivery is associated with delayed onset of lactation among urban Guatemalan women. Journal of Nutrition 2002; 132: 3055-3060

13 Haeri S, Dildy DA. Maternal mortality from hemorrhage. Seminars in Perinatology 2012; 36: 48-55

14 Hösli I, Gezer-Dickschat S. Postpartale Hämorrhagie. Hebamme.ch 2014; 9: 4

15 Hösli I, Vökt C, Lapaire O, Girard T, Gisin S (2014). Skript Geburtshilfliche Notfälle

16 Huch R, Schaefer R (2006). Iron Deficiency and Iron Deficiency Anemia. Stuttgart: Thieme

17 Mächler A, Wettstein S (2014). Postpartale Hämorrhagie nach Geburtseinleitung - Gefahr oder Mythos? Empfehlungen zur Überwachung des mütterlichen Blutverlustes nach einer Geburtseinleitung. Bachelorarbeit. ZHAW, Departement Gesundheit, Winterthur

18 Mehrabadi A, Hutcheteon JA, Lee L, Liston RM, Joseph KS. Trends in postpartum hemorrhage from 2000 to 2009: a population-based study. BMC Pregnancy Childbirth 2012; 12: 108

19 Meyer JW, Eichhorn KH, Vetter K, Christen S, Schleusner E, Klos A, Huch A, Huch R. Does recombinant human erythropoietin not only treat anemia but reduce postpartum (emotional) distress as well? Journal of Perinatal Medicine 1995; 23: 99-109

20 Mousa HA, Walkinshaw S. Major postpartum hemorrhage. Current Opinion in Obstetrics and Gynecology 2001; 13 : 595-603

21 Oyelese Y, Scorza WE, Mastrolia R, Smulian JC. Postpartum hemorrhage. Obstetrics \& Gynecology 2007; 34 (3): 421-441 
22 Rath W, Friese K (2009). Erkrankungen in der Schwangerschaft. Stuttgart: Thieme

23 Rath W, Bohlmann MK. Postpartale Hämorrhagie, Prävention und Therapie. Der Gynäkologe 2011; 44: 538-548. doi:10.1007/s00129-010-2739-6

24 Reynolds JL. Posttraumatic stress disorder after childbirth: the phenomenon of traumatic birth. Canadian Medical Association Journal 1997; 156: 831-835

25 Schlembach D, Mörtl MG, Girard T, Arzt W, Beinder E, Brezinka C, Chalubinski K, Fries D, Gogarten W, Hackelöer BJ, Helmer H, Henrich W, Hösli I, Husslein P, Kainer F, Lang U, Pfanner G, Rath W, Schleussner E, Steiner H, Surbek D, Zimmermann R. Management der postpartalen Blutung (PPH). Der Anaesthesist 2014; 63: 234-242. doi:10.1007/s00101-014-2291-1

26 Schrager S, Sabo L. Sheehan syndrome: a rare complication of postpartumhemorrhage. Journal of the American Board of Family Practice 2001; 14: 389-391

27 Sentilhes L, Gromez A, Clavier E, Resch B, Descamps P, Marpeau L. Long-term psychological impact of severe postpartum hemorrhage. Acta obstetrica et gynecologica Scandinavica 2011; 90 : 615-620. doi:10.1111/j.1600-0412.2011. 01119.x

28 Sheiner E (2011). Bleeding During Pregnancy. London: Springer

29 Snowdon C, Elbourne D, Forsey M, Alfirevic Z. Information-hungry and disempowered: a qualitative study of women and their partners' experiences of severe postpartum haemorrhage. Midwifery 2012; 28: 791-799

30 Surbek D, Irion O, Hess T, Drack G (2009). Expertenbrief No. 26. Kommission Qualitätssicherung. Präsident Prof. Dr. Daniel Surbek. Aktuelle Therapieoptionen der postpartalen Hämorrhagie (aktualisierte Version vom 3.11.2009)

31 Thompson JF, Heal LJ, Roberts CL, Ellwood DA. Women's breastfeeding experiences following a significant primary postpartum haemorrhage: A multicenter cohort study. International Breastfeeding Journal 2010; 5: 5

32 Thompson JF, Roberts CL, Ellwood DA. Emotional and physical health outcomes after significant primary post-partum haemorrhage (PPH): a multicenter cohort study. The Australian \& New Zealand journal of obstetrics \& gynaecology 2011; 51: 365-371. doi:10.1111/j.1479-828X.2011.01317.x
33 Thompson JF, Ford B, Raynes-Greenow CH, Roberts CL, Ellwood DA. Women's Experiences of Care and Their Concerns and Needs Following a Significant Primary Postpartum Hemorrhage. Birth: Issues in Perinatal Care 2011; 38 (4): 327-335

34 WHO (2012). WHO recommendations for the prevention and treatment of postpartum haemorrhage. Geneva: World Health Organisation. Heruntergeladen von http://apps.who.int/iris/ bitstream/10665/75411/1/ 9789241548502_eng.pdf am 14.02.2015

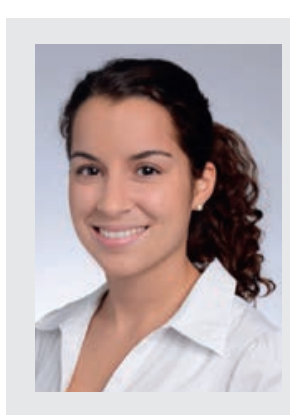

Ines Neukom

Hebamme, BSc

Zürich inesneukom@hotmail.com 